\title{
46th Winter School on Wave and Quantum Acoustics XLVth Jubilee Winter School on Environmental Acoustics and Vibroacoustics Szczyrk, Poland, February 27-March 2, 2017
}

On behalf of Upper Silesian Division of the Polish Acoustical Society (main organizer) and Institute of Physics - Centre for Science and Education at the Silesian University of Technology and Committee of Acoustics of the Polish Academy of Sciences (coorganizers) we are pleased to announce that it is nearing the end of preparations for the conduct traditionally organized Conferences, which will take place as previously in Szczyrk. At the moment, more than 50 people have confirmed participation in Conferences. They will present approximately 40 lectures and reports.

46th Winter School on Wave and Quantum Acoustics constitutes a platform for sharing the results and achievements obtained in different branches of physical acoustics (e.g. molecular acoustics, quantum acoustics, acousto-optics, magnetoacoustics, photoacoustics, acoustics of solid state etc.). Moreover, researches in some selected topics related to those mentioned above (e.g. optoelectronics, relaxation processes) will also be presented during the school. Currently, during the Conference Workshop on Acoustoelectronics and Workshop on Molecular Acoustics, Relaxation and Calorimetric Methods will be held. However, there is a possibility of organizing workshops on other subjects in the future. We would like to invite scientific centers and groups to cooperate in organizing workshops on the subjects of their interests.

XLVth Jubilee Winter School on Environmental Acoustics and Vibroacoustics is the forum for all environmental and vibroacoustics fields. Particularly it concerns traffic noise, vibroacoustics of machines, room acoustics, building acoustics, noise protection and similar problems. During the Conference in association with SVANTEK there will be organised seminars on "Measurement of noise and vibration at the workplace", "Enivironmental noise monitoring" combined with measurement workshops.
The Conferences will begin with a special session dedicated to the memory of Professor Eugeniusz Danicki.

In this issue one can find abstracts of some lectures, which will be presented during the Conferences. Further information about Conferences is available on our website

$$
\text { http://ogpta.pl/index.php/oddzial/ }
$$

Chairman of Organizing Committee Franciszek Witos

Abstracts

\section{3th Winter Workshop on Acoustoelectronics}

Using Clustering Methods for the Identification of Acoustic Emission Signals Generated by the Selected Form of Partial Discharge in Oil-Paper Insulation

BoRUCKI Sebastian, s.borucki@po.opole.pl

ŁUCZAK Jacek

ZMARZŁY Dariusz

Institute of Electrical Power and Renewable Energy Opole University of Technology

Prószkowska 76, 45-758 Opole, Poland

The article presents the results of the use of clustering methods (cluster analysis) to identify the signals of acoustic emission (AE) generated by partial discharge (PD) in the paper-oil insulation. As part of the research qualitative analysis the following clustering methods of the registered AE signals were used: method of single bond (Single linkage), the full bond method (Complete linkage), the secondary connections method (Average linkage), the centroid method (Centroid linkage) and Ward's method (Ward linkage). The objective of the analysis was to find a test measurement of the series AE signals, derived from the dif- 
ferent forms of PD elements, forming the aggregates (clusters), where in terms of a particular characteristic or established criteria for the components are as similar as possible and at the same time up other than in the other groups. Then, on the basis of clustering attempted, the evaluation of the effectiveness identification of the particular PD forms generated in power transformer paper-oil insulation system was made. Appropriate analyzes and simulations were performed using the computing environment Matlab and available in this environment of the clustering procedures. As part of the study analyzed the results of series AE signals generated of the basic PD forms, which were obtained in laboratory conditions using spark gaps modeling the defects of the power transformers insulation systems.

\section{Characterization of Acoustic Emission Signals Generated by Partial Discharges in High Voltage Current Transformers}

\author{
Cichoń Andrzej, a.cichon@po.opole.pl \\ Faculty of Electrical Engineering, Automatic Control \\ and Computer Science \\ Opole University of Technology \\ Prószkowska 76, 45-758 Opole, Poland
}

High voltage current transformers (HVCT) are commonly used apparatus in electrical power distribution systems. HVCT insulation system design is quite complex. High voltage and grounded parts are separated by porcelain insulation. Inside of the porcelain there are windings covered with an oil paper insulation, furthermore a proper electric field distribution is provided by some additionally mounted electrodes. Due to insulation system ageing process some degradation of dielectrics may occur which usually leads to local partial discharge (PD) generation. Various combustible gasses production is accompanied by PD generation as well as a significant pressure increase may also be noticed inside of a HVCT. In some exceptional circumstances an internal pressure may exceed a critical value and a porcelain insulator may explode thus a fire of the entire insulation system is also possible.

Laboratory measurement results on the J110-4a type HVCT with nominal voltage of $110 \mathrm{kV}$ are presented in the paper. A severe insulation system fault is detected during research. An acoustic emission (AE) method is applied for PD source detection and localization measurements. Some exemplary AE signals generated by internal PDs during various supply voltage levels are registered by wideband piezoelectric AE joint sensor mounted on the porcelain insulator surface. Selected fundamental descriptors of $\mathrm{AE}$ signals in time, frequency and time-frequency domain are applied for detailed source signals characterization. Apart from $\mathrm{AE}$ measurements, additionally an electrical method PD measurements are proceeded simultaneously. Finally, presented results may also be taken as a benchmark for further measurements on apparatus under normal on-site operation conditions.

\section{Seismometer Based}

\section{on Surface Acoustic Waves Transducers}

FiLIPIAK Jerzy, filipiak1947@gmail.com

Institute of Electronic and Control Systems

Technical University of Czestochowa

Al. Armii Krajowej 17, 02-240 Częstochowa, Poland

The paper will be presented the concept of a seismometer based on surface acoustic waves (SAW). The base of the system design are three SAW transducers arranged along three orthogonal axes. Each transducer is composed of two SAW delay lines. The seismometer consists of high frequency generator, phase detector and three SAW transducers. It allows to measure constant and time dependent accelerations in three orthogonal directions with the operating band up to $150 \mathrm{~Hz}$. We will show an analysis of main system parameters i.e. amplitude frequency response, resonant frequency and sensitivity including cross-sensitivity. We will discuss temperature influence on these parameters, also. We will present experimental results of the seismometer transducer and compare them with theory.

Currently used seismometers in seismology, mine seismology and reflection seismology are design based on system of mechanic pendulums. We will compare these systems with presented above in terms of technical performance, prices and installations.

\section{$\star \star \star$}

Comparative Analysis of the Results

of Snowfall Level Measurements Performed

Using Ultrasonic Aerolocation Method

in Real Conditions

in Different Climatic Areas

Gudra Tadeusz, Tadeusz.Gudra@pwr.edu.pl

BANASIAK Dariusz

HERMAN Krzysztof

OPIELIŃski Krzysztof

Faculty of Electronics

Wrocław University of Science and Technology

Wybrzeże Wyspiańskiego 27, 50-370 Wrocław, Poland

This paper addresses the problem related to the interpretation of the results of actual snowfall measurements performed with two different configurations of the compensation methods for changeable climatic conditions. The paper also presents the operating principle of an ultrasound sensor for monitoring snow layer (on the air-side). In particular, the paper includes a graphic representation of the results of measurements performed in measurement stations located in Poland and at Spitsbergen. The two compensation methods (temperature and parametric) were tested for their effectiveness in different, changeable climatic conditions. The measurement results confirm that the applied compensation methods are useful in minimizing the measurement error for snow layer thickness measured on the air-side. 


\section{Experimental and Numerical Analysis of the Response in Non-Steady Response Step and Steady Stage of a SAW Structure with PANI+Nafion on Action of Carbon Monoxide}

HEJCZYK Tomasz, fundacja.ark@gmail.com

Fundacja Akademia Rozwoju Kreatywnego Wyzwolenia 117, 44-321 Marklowice, Poland

The paper presents the results of numerical analyses of the SAW gas sensor in the steady and non-steady in response state. The effect of SAW velocity changes vs. the surface electrical conductivity of the sensing layer is predicted. The conductivity of the porous sensing layer above the piezoelectric waveguide depends on the profile of the diffused gas molecule concentration inside the layer.

Numerical results for the gas CO for layer (Polyaniline) PANI+Nafion in the steady state and non-steady state in response step have been shown. The main aim of the investigations was to study thin film interaction with target gases in the SAW sensor configuration based on diffusion equation for polymers. Numerical results for profile concentration of the response in non-steady state use Finite Difference method have been shown.

The results of numerical analyzes allow to select the sensor design conditions, including the morphology of the sensor layer, its thickness, operating temperature and layer type. The numerical results basing on the code written in Python, are described and analyzed. The theoretical results were verified and confirmed experimentally.

$$
\star \star \star
$$

\section{Low Temperature Catalytic Hydrogen SAW Sensor}

JASEK Krzysztof ${ }^{1}$, krzysztof.jasek@wat.edu.pl

PAsternak Mateusz ${ }^{2}$, GraBKA Michał ${ }^{2}$

1 Institute of Chemistry

2 Institute of Radioelectronics

Military University of Technology

S. Kaliskiego 2, 00-908 Warszawa, Poland

Hydrogen is used as a reducing agent and a carrier gas in the various technological processes. It is also known as an ecologic fuel and highly explosive gas. For this reasons the need for constructing a sensor for fast, selective and highly sensitive hydrogen detection at room or near room temperature is still topical. A number of different kinds of hydrogen sensors have already been developed. The devices are based on various physical phenomena; hydrogen particles change mechanical, thermal, electrical, optical or acoustical properties of the detector.

In the paper a new kind of sensitive film for surface acoustic wave (SAW) hydrogen sensing is proposed. The film is based on Nafion ${ }^{\circledR}$ matrix containing electrosprayed platinum particles. It utilises catalytic hydrogen oxidation; water produced as a result of this process is absorbed via Nafion sulfonate groups and this causes both mechanical and electrical changes of film properties influencing the SAW parameters.

A laboratory model of sensor was fabricated based on two-port resonator structure on quartz STX substrate working at operating frequency of about $197 \mathrm{MHz}$ and electrosprayed thin Nafion/Pt film. The device was tested using a dedicated electronic system and a gas chamber with temperature and gas-flow control. The obtained results show that at temperatures over $40^{\circ} \mathrm{C}$ the sensor is highly sensitive to low concentration of hydrogen and it exhibits very fast response, good sensitivity and short-term repeatability.

$$
\star \star \star
$$

\section{Ultrasonic Welding Generator for Frequency Range from 16 to $60 \mathrm{kHz}$}

KARDYŚ Witold, witold.kardys@itr.org.pl MiLewski Andrzej

Tele and Radio Research Institute Ratuszowa 11, 03-450 Warszawa, Poland

The paper presents high power ultrasonic generator for welding and cutting processes developed by Tele \& Radio Research Institute. The device is used for manufacturing various products such as: PE pipes with aluminum insert, battery packs, car components, trowels, dust masks, corner beads and many other. The new generator can provide up to $5 \mathrm{~kW}$ of electrical power to ultrasonic transducer in frequency range from $16 \mathrm{kHz}$ up to $60 \mathrm{kHz}$ with regulation step down to $0.1 \mathrm{~Hz}$. The device utilizes innovative microcontroller with built-in high resolution timing blocks that enable direct synthesis of control signals for generator's resonant converter without the need of external DDS unit or programmable device. This new approach to designing ultrasonic generators can benefit in greater flexibility and reliability of the device. New algorithms with cycle by cycle parameter control and precise regulation of output frequency and power delivery have been developed. Various parameters of ultrasonic stack such as impedance and resonant frequency are measured by the generator in real time and can be used to diagnose the stack and detect its damages.

\section{$\star \star \star$}

\section{An advanced Technologies}

\section{for Radar Signal Processing - the Review}

KAWALEC Adam, adam.kawalec@wat.edu.pl

Institute of Radioelectronics

Military University of Technology

S. Kaliskiego 2, 00-908 Warszawa, Poland

The selected applications of the surface acoustic wave devices in the area of the signal processing are presented. The analysis of the surface acoustic wave (SAW) filter is described in the paper. In the analysis based on the spectral theory is taken into account. The analysis of the effective surface permittivity significantly simplifies the theoretical analysis of the interdigital transducer. The calculation of the complex effective surface permittivity can be used to estimate the band of the possible (undesirable) bulk waves. The synthesis of the dispersive line based on the theoretical analysis results is presented. The experimental characteristics for the quartz based dispersive line as an application for the radar signal processing is demonstrated. Dispersive delay lines can be applied in chirp pulse compression system characterized low side-lobe level and in SAW spectrum analyser as well.

The paper is dedicated to the memory of Professor Eugeniusz Danicki. 
Application of a Phase Resolved Partial Discharge Pattern Analysis for Acoustic Emission Method in High Voltage Insulation Systems Diagnostics Study on Descriptors of Acoustic Emission Signals Generated by Partial Discharges Under Laboratory Conditions and in On-Site Electrical Power Transformer

Kunicki Michał, m.kunicki@po.opole.pl, CichoŃ Andrzej

Faculty of Electrical Engineering, Automatic Control and Computer Science

Opole University of Technology

Prószkowska 76, 45-758 Opole, Poland

An acoustic emission (AE) method is widespread and willingly applied in high voltage insulation systems diagnostics, especially in fields of partial discharge (PD) source detection and localization. According to contemporary applied techniques, an alternative method for PD generated AE signals analysis based on a correlation between voltage phase run and AE pulses, so-called phase resolved $\mathrm{PD}$ pattern (PRPD), is presented in the paper. PRPD pattern is a well-known analysis tool commonly used in such PD diagnostic methods as conventional electrical method as well as UHF method. Moreover it yields various signal analysis abilities and allows a direct correlation indication between measurement results achieved using different methods. An original PRPD measurement methodology applied for AE method as well as some exemplary measurement results and further data analysis capabilities are announced in the paper. Also a comparative analysis of PRPD patterns achieved using various measurement methods and different $\mathrm{PD}$ source configurations is investigated in the research. All presented experiments are proceeded under laboratory conditions.

The main purpose of the presented research is to indicate an all-embracing analytical tool that yields an ability to $\mathrm{AE}$ measurement results direct comparison (qualitative as well as quantitative) with other commonly applied PD measuring methods. Presented results give a solid fundamental for further research works concerned with a direct correlation method for $\mathrm{AE}$ and other described in the paper diagnostic methods, mainly in order to PD phenomena analysis and assessment in real-life high voltage apparatus insulation systems under normal on-site operation conditions.

\section{Prototype of Wideband Ultrasonic Sonar Based on STM32}

OKOŃ-FĄFARA Marta, marta.okon@wat.edu.pl KAWALEC Adam, FĄFARA Bartłomiej

Institute of Radioelectronics

Military University of Technology

S. Kaliskiego 2, 00-908 Warszawa, Poland

The paper presents a project of a low-cost prototype version of an ultrasonic wideband sonar and the problems occurring during the development process. The aim of this work was to design a short range sonar capable of the transmission of an arbitrary shape wideband signal. The chosen transmitting sensor typically works in pulsed operation modes. The dedicated output circuits had to be designed to expand its capabilities without information losses.
The transmission and reception of the signals is managed by a Discovery evaluation board with a STM32 microcontroller. The receiving data are saved on a SD card and processed of-line.

$$
\star \star \star
$$

\section{Acoustic Emission Testing of Storage Tanks}

OLszewska Aneta, aneta.olszewska@polsl.pl

Department of Optoelectronics Faculty of Electrical Engineering Silesian University of Technology Krzywoustego 2, 44-100 Gliwice, Poland

The paper presents the use of the acoustic emission (AE) method for diagnostic testing of above ground storage tanks (AST). This method allows evaluation and location of active corrosion damages of bottom material and micro leaks in the operating conditions of the tested object. To prepare object for AE testing it is not necessary to drain the storage medium from tested tank, which provides a high state of security at significant diagnosis and operation cost savings.

The methodology and AE test results carried out on the three storage tanks of crude oil are presented. Recorded during the measurements data have been analyzed. The conclusions drawn from this analysis, give the location of the $\mathrm{AE}$ sources and the evaluation of the integrity of the studied tanks bottom. Additionally, the effects of external conditions (sunlight and wind) on the obtained results are shown.

Moreover, evaluation criteria of technical condition of the tanks based on acoustic emission signals are presented, on the basis of which evaluation of the state of the tested tanks was carried out and operating time for the next test has been estimated.

\section{$\star \star \star$}

Ultrasound Tomography: Results of Breast Phantom Imaging

OPIELIŃsKi Krzysztof J. ${ }^{1}$, krzysztof.opielinski@pwr.edu.pl, Pruchnicki Piotr ${ }^{1}$, Wrzosek Marcin ${ }^{2}$, Nicpoń Józef ${ }^{2,3}$, PODGÓRSKI Przemysław ${ }^{4}$

${ }^{1}$ Faculty of Electronics

Wrocław University of Science and Technology

Wybrzeże Wyspiańskiego 27, 50-370 Wrocław, Poland

2 Department of Internal Diseases with Clinic

for Diseases of Horses Dogs and Cats

Faculty of Veterinary Medicine

Wrocław University of Environmental and Life Sciences Plac Grunwaldzki 47, 50-366, Wrocław, Poland

${ }^{3}$ Centre for Experimental Diagnostics

and Biomedical Innovations of the Wrocław University of Environmental and Life Sciences

Plac Grunwaldzki 47, 50-366, Wrocław, Poland

${ }^{4}$ Department of General and Interventional Radiology and Neuroradiology

Wrocław Medical University

Borowska 213, 50-556 Wrocław, Poland

In order to improve breast cancer detection rates, new and better imaging methods are required. Currently, the ultrasound tomography as non-invasive and safe hybrid method may contribute to achieving a new standard for 
breast cancer diagnostics. The aim of the paper was to analyze the imaging ability of tissue-like media structure found in female breast using the developed novel ultrasound computer-assisted tomographic scanner. Measurements was performed on commercial breast biopsy phantoms due to their well defined structure with inclusions mimicking glandular tissue with lesions. Obtained magnetic resonance images of the same phantoms were used for comparison as the gold standard.

$$
\star \star \star
$$

Determination of Some Kinetic Parameters of Fast Surface States in Semiconductors by Means of the Surface Acoustic Wave Method

Pustelny Tadeusz, tpustelny@polsl.pl

Department of Optoelectronics

Silesian University of Technology

Krzywoustego 2, 44-100 Gliwice, Poland

Among the methods of investigations of semiconductor surfaces, there are no methods of investigating the kinetic properties of electrical carries in fast and very fast surface states. The existing methods allow only investigations of the surface sates with a carrier life-time $\tau$ of above $10^{-8} \mathrm{~s}$. In the case of extrinsic semiconductors the surface states may, however, be considerably faster (the carrier life-time in surface traps is usually less than $10^{-8} \mathrm{~s}$ ). In such cases the existing methods of determining the parameters of fast surface states allow only to estimate these parameters, since the obtained results exhibit a considerable uncertainty. For this reason, investigations of the kinetic properties of fast surface states are not popular and there aren't any new results concerning their determination.

For some years attention has been paid to the influence of the physical state of the near-surface region of a semiconductor on the results of investigations of the acoustoelectric effects in piezoelectric-semiconductor systems. The attention has been paid to the possibility of applying Rayleigh's surface acoustic waves SAWs for investigations of various surface parameters of solid states.

The theoretical and experimental results of the application of acoustoelectric effects (longitudinal and transverse) for the determination of carrier properties in near surface region (e.g. the surface electrical potential, carrier concentration, electrical conductivities,... ) have been presented. Problems connected with the determination of the chemical and mechanical means of surface treatments in the first step of preparation of semiconductor plates for technology on their kinetic properties have not often been taken up. The quantitative data concerning the effective life-time $\tau$ and the velocity of carrier trapping $g$ are very seldom presented in literatures of semiconductor surfaces. Presented results are new and original.

\section{$\star \star \star$}

\section{Project of SODAR with Phased Array Antenna}

Rogala Tomasz, tomasz.rogala@wat.edu.pl KAWALEC Adam

Institute of Radioelectronics

Military University of Technology

S. Kaliskiego 2, 00-908 Warszawa, Poland

This article is dedicated to the presentation of the project of remote sensing device, which is SODAR (Sound
Detection And Ranging). In this article will be presented theoretical knowledge of work rules of SODAR there will be also described previous achievements and solutions to be implemented in the future.

SODAR is a remote sensing device, which by usage of sound waves allows to measure parameters such as altitude, speed and direction of the wind. SODAR operates in pulse mode. That is, in the atmosphere is transmitted short pulse probe, followed by a echo listening phase. The project of SODAR involves the use of phased array antenna in the form of a matrix of sound transmitters, which allows digital beam forming. The transmitted signal is a signal with internal modulation or manipulation of frequency which aims to increase its bandwidth.

So far, there were made some prototype components of the apparatus. One of the first prototypes was the transmit path. The purpose of this test was to check the capabilities of powering piezoelectric transducers via integrated audio power amplifier. The indirect aim was also to examine the mechanical strength of the transmitters. The next step was to develop the receiving track. It was based on the use of integrated microphone amplifiers MAX9814. Each of the designed transceiving modules are based on STM32F407 microcontroller.

Current work focuses on receiving and registering the actual echo signal. Sampled echo signal is used for research on signal processing algorithms and to design the shape of the transmitted signal. Activities carried out so far show wide opportunities for further development of the project, as evidenced by increasingly favorable results of research and testing.

\section{A Wideband Ultrasonic Imaging Sonar System}

Serafin Piotr, piotr.serafin@wat.edu.pl

OKON-FAFARA Marta

Szugajew Marcin

LESNIK Czeslaw

KAWALEC Adam

Institute of Radioelectronics

Military University of Technology

S. Kaliskiego 2, 00-908 Warszawa, Poland

Acoustic echolocation systems provide information on distance to observed objects. However, typically the ultrasonic transducers posses relatively wide transmitting or receiving patterns, which does not allow to achieve high resolution images of the objects in the cross range direction.

In synthetic aperture sonar (SAS) systems the acoustic sensor is mounted on a platform that in classical configuration moves perpendicularly to the observation direction. The sensor emits the sounding signals, receives the echo signals along the platform's trajectory and stores them in its memory. The signals are subsequently processed as if they were received by a long antenna which allows to obtain very high cross-range resolution images that is comparable with used wavelength. The range resolution of the image depends on the bandwidth of the transmitted signal.

The paper presents an experimental high resolution imaging acoustic system based on a prototype wideband ultrasonic sonar sensor. The results of the system's performance are presented.

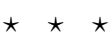




\section{Application of the Signal Synthesizer} for SODAR System

STEC Olga, olga.stec@student.wat.edu.pl

Rogala Tomasz, Kawalec Adam

Institute of Radioelectronics

Military University of Technology

S. Kaliskiego 2, 00-908 Warszawa, Poland

SODAR (Sound Detection And Ranging) is a remote sensing, pulse operating device, which by using of echo from emitted sound waves investigates the distribution and movement of turbulence in the atmosphere. This article is devoted to the project of signal synthesizer, which has a wide range of applications and is characterized by its flexibility in adjusting parameters of generation. The synthesizer is provided to cooperate with the amplifier as a probe signal generator. This combination will be placed in each of the independent transmit-receive modules in designed SODAR.

The main module of the whole synthesizer is STM32F407 microcontroller, whose task will be to generate a signal in the hearing range with 12 bits resolution of amplitude. Properly prepared signal samples will be stored in FLASH memory. They can be read out in accordance with a predetermined scenario, which will result in a different frequency or modulation of the signal, for example linear frequency modulation (LFM). Beside of the signal output connected to the amplifier microcontroller has two controlling ports: transmission start and receive start. Each of the independent transmitting modules will be controlled by an external signal of the start of the transmission. As a result, a set of modules placed side-by-side will form the matrix of sound transducers. This kind of structure and control will allow to apply digital beam forming. Additional functionality of the synthesizer system will be generating test signals to identify the efficiency of the individual elements of the transmit path.

The synthesizer is part of SODAR system which is still developed and its concept is subject of constant modifications. A microcontroller as a signal synthesizer can in the future be expanded with new functionalities as the data acquisition. Activities carried out so far show a wide opportunities for further development of the project.

\section{Application of ADC and ADP Descriptors to Identify the Acoustic Emission Signals Generated by the Partial Discharges}

SzERsZEŃ Grzegorz ${ }^{1}$, szerszen_g@yahoo.com Witos Franciszek ${ }^{2}$, franciszek.witos@polsl.pl

${ }^{1}$ Department of Electronics and Telecommunications State Higher Vocational School in Tarnow

Mickiewicza 8, 33-100 Tarnów, Poland

${ }^{2}$ Department of Optoelectronics

Faculty of Electrical Engineering

Silesian University of Technology

Krzywoustego 2, 44-100 Gliwice, Poland

In this paper, there are presented the research results of partial discharge in the systems with modeled sources, carried out by calibrated acoustic emission method. Within the method named as the calibrated acoustic emission method, measurements are carried out simultaneously by means of two methods i.e. acoustic emission and electric. Finally the results of partial discharge research obtained by means of acoustic emission method are refered to results of measurements of apparent charge introduced by the acting source of partial discharge (obtained by means of electric method).

There are described designed and constructed modeled sources of partial discharge and the measurement stand consisting of a vat of oil with mounted modeled sources, the test set for generating high voltage, author measurement system 8AE-PD (acoustic emission method) and system TE571 (electric method). Research methodology is presented, too.

For the recorded signals, phase-time characteristics, averaged phase characteristics, averaged Short-Time Fourier Transform spectrograms and the frequency characteristics were calculated. Basing on calculated characteristics, there were carried out qualitative analysis of signals registered in the measurement channels with wideband and resonant sensors and quantitative analysis of the selected signal parameters, in particular the authors descriptors of acronyms $\mathrm{ADC}$ and ADP.

Noise signals were compared with signals generated by partial discharge. Analysis showed the possibility of distinguishing of signals generated by partial discharge and noise. Description of signals using of ADP and ADC descriptors showed additional capabilities to confirm the presence partial discharge sources within the examined object.

$$
\star \star \star
$$

\section{Simulation of Infrasound Waves Emitted by wind Turbine}

WotzKa Daria, d.wotzka@po.opole.pl

Institute of Electric Power and Renewable Technologies Opole University of Technology

Prószkowska 76, 45-758 Opole, Poland

The subject matter considers generation and propagation of infrasound waves emitted by wind turbines and in particular with their simulation using computational software. Nowadays computers and software tools enable for integration of existing equations, describing physical phenomena from various fields, including mechanics, acoustics and aerodynamics into one multiphysical numerical model and to analyze the model dynamics in time and space. The presented results are related to development of the computer model of a wind turbine, with adequately defined parameters, for to enable study of low-frequency acoustic wave generation and propagation in the near and far field. The model integrates various physical phenomena responsible for the generation of low frequency noise by wind turbines, into a single model. Theoretical analysis of the impact of weather conditions (wind speed and direction, temperature, humidity, atmospheric pressure) and the topography of the land surrounding the wind turbine (type of ground, absorption and reflection from the surface, shielding by obstacles) on the path of propagation (trajectory) of low-frequency acoustic waves is presented and discussed. 


\section{3th Winter Workshop on Molecular Acoustics, Relaxation and Calorimetric Methods}

\section{Conductance and Ionic Association of Imidazolium Ionic Liquids in Alcohols}

Boruń Agnieszka, chmielewska.a@gmail.com BALD Adam

Department of Physical Chemistry

University of Łódź

Pomorska 163, 90-236 Łódź, Poland

Physical properties of solvents such as density, viscosity, relative permittivity, and the capability of hydrogen bond formation as well as the ion-solvent and solventsolvent interactions influence the conductance behavior of electrolytes in solutions. Ion-solvent interactions stabilize the ion by solvating it, which affects the ionic association. Therefore, in order to investigate the effect of the nature of the ions, the structure of the solvent medium or the relative permittivity on the solvation and ionic association, the conductivity measurements are often used.

We present the conductometric properties of dilute solutions of $[\mathrm{emim}][\mathrm{BF} 4]$ and $[\mathrm{bmim}][\mathrm{BF} 4]$ in methanol, propanol, propan-2-ol and butanol over a wide temperature range. The ILs show moderate ion association in methanol and strong in other alcohols. The values of limiting molar conductivities for the ILs in alcohols generally follow the order: $\mathrm{MeOH}>\mathrm{PrOH}>2-\mathrm{PrOH}>\mathrm{BuOH}$, which correlates well with decrease in viscosity of the solvent. In turn, the association constant values change in the reverse order than $\Lambda$ o values, which correlates well with an increase in relative permittivity of the solvent. The thermodynamics of the association process were also evaluated and discussed.

High Pressure Acoustic and Thermodynamic Properties of 2-Methylfuran + Isooctane, the Second Generation Biofuel Blends. Experiments and PFP Modeling

DzIDA Marzena, mhd@ich.us.edu.pl JĘŻAK Sylwia

Institute of Chemistry

University of Silesia

Szkolna 9, 40-006 Katowice, Poland

The 2-methylfuran can be obtained from cellulosic biomass and is indicated as a potential bioethanol or petroleum-based fuels substitute. As a blending component for 2-methylfuran, isooctane (2,2,4-trimethylpentane) was chosen. Isooctane is used as a model fuel in a wide range of applications; it even serves as the reference standard for the "Research Octane Number". Optimization of the fuel injection process requires knowledge of densities, speeds of sound, heat capacities, isobaric thermal expansions, isentropic and isothermal compressibilities. A thermophysical study is important to understand properties depending on intermolecular interactions and the structure of chemicals. Thus, the first objective of this work is to examine the influence of the temperature and pressure on the abovementioned properties as well as on excess volumes, ex- cess isentropic and isothermal compressibilities, excess isobaric thermal expansions, and excess heat capacities of 2methylfuran + isooctane mixtures. The next objective is to study intermolecular interactions and structure of the mixtures under test basing on the Prigogine-Flory-Patterson (PFP) theory.

$$
\star \star \star
$$

Structure - Property Relationship for Surface Tension of Task Specific Ionic Liquids

Geppert-Rybczyńska Monika, monika.geppert-rybczynska@us.edu.pl

Institute of Chemistry

University of Silesia

Szkolna 9, 40-006 Katowice, Poland

In this work, the surface tension, sigma, of chosen Ionic Liquids (ILs) is presented. Substances analyzed here contain some substituents in cation, or have a kind of anion that provide task specific functionality such as chirality, or activity against bacteria or fungi, and others. They are regarded homologues series of Ionic Liquids or ILs with groups, which influence the symmetry of cation. The relation between surface tension and ions structure in homologues series is discussed and compared with those found for the other most popular ILs homologues series: 1-alkyl3-methylimidazolium tetrafluoroborates, [CnC1im][BF4], bis(trifluoromethyl-sulfonyl)imides, [CnClim][NTf2] and hexafluorophosphates, [CnC1im][PF6]. A parachor concept is applied for "sigma" prediction assuming additivity in relation to constitution of ions. Despite of complex structure of investigated substances surface tensions calculated in this work are in agreement with experimental values.

$$
\star \star \star
$$

\section{Heating Induced by Therapeutic Ultrasound in the Presence of Magnetic Nanoparticles}

KaCzMarek Katarzyna, Hornowski Tomasz JózEFCZAK Arkadiusz, aras@amu.edu.pl

Institute of Acoustics

Faculty of Physics

Adam Mickiewicz University

Umultowska 85, 61-614 Poznań, Poland

Ultrasound (US) can be used for biomedical applications such as imaging and therapy. One of the earliest medical applications of ultrasound is the therapeutic heating of tissue. The effectiveness of ultrasound in hyperthermia therapy can be significantly improved by using the so-called sonosensitizers dispersed in heating tissue. Many different inorganic and organic substances can be used as sonosensitizer which can maximize the effect of ultrasonic irradiation, e.g. superparamagnetic iron oxide nanoparticles (SPIONs) with mean sizes of 10-300 nm. The objective of this study is to assess the utility of using magnetite nanoparticles during ultrasound hyperthermia to enhance heating at low US power. The effect of SPION concentration on heating rate was investigated in a tissue-mimicking phantom using an ultrasound system with planar transducer. The 
influence of penetration depth on temperature increase was also studied experimentally and theoretically.

This work was supported by a Polish National Science Centre grant, no DEC-2015/17/B/STr/03566.

$$
\star \star \star
$$

\section{The Enhancement of the Thermal Effect}

\section{in Hyperthermia Due to Nanoparticle Application}

KACZMAREK Katarzyna ${ }^{1}$, aras@amu.edu.pl JózEFCZAK Arkadiusz ${ }^{1}$, Hornowski Tomasz ${ }^{1}$ KubovČíková M. ${ }^{2}$, SKumiel A. ${ }^{1}$

Rozynek Z. ${ }^{1}$, Timko M. ${ }^{2}$

${ }^{1}$ Institute of Acoustics

Faculty of Physics

Adam Mickiewicz University

Umultowska 85, 61-614 Poznań, Poland

${ }^{2}$ Institute of Experimental Physics

Slovak Academy of Sciences

Watsonova 47, 04001 Košice, Slovakia

Ultrasonic and magnetic hyperthermia are very popular medical procedures used nowadays in many cancer treatments. Both of these methods cause a temperature rise of cancer cells. As a result of the temperature increase, cancer cells are weakened or destroyed.

In our study we conducted both type of hyperthermia treatments on agar phantoms which mimic soft tissues of the human body. In the first part, we studied the influence of magnetic fluid on the thermal effect of ultrasonic hyperthermia. Theoretically, the addition of scattering material like nanomagnetic particles can lead to additional heat due to supplementary scattering. In the second part, we investigated magnetic hyperthermia with use of the same magnetic fluid as previously. We also conducted a synergic experiment with a combination of both types of hyperthermia. The obtained results confirm theoretical assumptions that the addition of scattering material can enhance ultrasonic hyperthermia.

This work was supported by a Polish National Science Centre grant, no DEC-2015/17/B/ST7/03566.

The Application of a Four Segment Composition Model for Excess Molar Volume and Excess Compressibility Coefficient of 2-Alkoxyethanols - Water Mixtures in a Wide Temperature Range

KLIMASZEWSKI Krzysztof, krzyklim@poczta.wp.pl BALD Adam

Department of Physical Chemistry

University of Łódź

Pomorska 163, 90-236 Łódź, Poland

The course of excess molar volume and compressibility factor can be described using Redlich-Kister's or Ott's equations. For more complex curve courses describing with one equation for the entire range of concentrations becomes impossible. Presentation shows the application of four-segment structure model of a mixed solvent prepared by Douheret, Davis and Høiland to describe excess molar volume and excess compressibility coefficient of mixtures of 2-alkoxyalkohols with water over a wide temperature range. The coverage analysis of the different areas (water-rich, pseudolamellar, transitional and organic-rich segment) for homologous series alkoxyalkohols was conducted over wide temperature range.

\section{Physico-chemical Characterization of Magnetoferritin and its Potential Bio-Applications}

KopCANsky Peter, kopcan@saske.sk

Institute of Experimental Physics

Slovak Academy of Sciences

Watsonova 47, 04001 Košice, Slovakia

Magnetoferritin (MFer) is biological complex composed of protein shell, apoferritin, and synthetically prepared magnetic iron oxides inside. Due to ist unique properties (superparamagnetic behavior, nanoscale size and biological origin) it is believed to have various biomedical application (e.g. a drug carrier in the targeted transport). Small Angle X-ray (SAXS) and Neutron (SANS) Scattering studies have shown partial shell destruction of MFer and increasing of polydispersity with the loading factor (LF: average number of iron atoms per one complex) growth. Presence of magnetic nanoparticles in MFer could play an important role at observed ability to reduce the size and amount of lysozyme amyloid fibrils (LA), confirmed by SAXS and fluorescence measurements. Next investigations using UV-VIS spectrophotometry have confirmed peroxidase-like activity of MFer. Preliminary calorimetric measurements have shown the increase in temperature during exposition of MFer in AC magnetic field during magnetic hyperthermia tests. Presence of magnetic nanoparticles in MFer affects also the contrast at Magnetic Resonance Imaging (MRI), that could be useful for diagnosis associated with magnetic nanoparticles formation in damaged ferritin at neurodegenerative or cancer diseases.

$$
\star \star \star
$$

\section{Relaxation in Several Derivatives of Benzene}

LiNDE Bogumił, fizbl@univ.gda.pl

Institute of Experimental Physics

University of Gdańsk

Wita Stwosza 57, 80-309 Gdańsk, Poland

Acoustical spectroscopy at frequencies up to $10 \mathrm{GHz}$ gives the possibility of the investigation of liquid substances, where the relaxation process observed is caused by energy transfer between the degrees of freedom.

The presented results of research in five halides of benzene as well as toluene and aniline are discussed and compared to benzene. Based on investigations by the author as well as by other people, and taking into account experimental and literature data concerning a great number of compounds, one can draw a conclusion that almost all acoustic relaxation processes in liquids can be described using a single relaxation time. It also seems that all vibrational degrees of the molecule take part in this process. 
It is known that the appearance of differences in transition probabilities could be caused by additional attraction in interactions of molecules having dipole moments. Halides have higher values of dipole moments than benzene. This difference could be responsible for the difference of transition probabilities and changes in the relaxation times.

However, benzene derivatives with amino, nitro, and methyl groups and halides show the other type of relaxation.

\section{Structure and Hyperthermia Characterization of Bacterially Synthesized Magnetic Nanoparticles}

Timko Milan, timko@saske.sk

Institute of Experimental Physics

Slovak Academy of Sciences

Watsonova 47, 04001 Košice, Slovakia

The magnetosomes were prepared by biomineralization process of magnetotactic bacteria Magnetospirillum sp.AMB-1 with specific length depending on the number of individual nanoparticles found in the chain. Structure characterization of magnetosome chains after isolation (IM) from bacteria and after treatment isolated sample by sonication effect (SM) was done by dynamic light scattering (DLS), small-angle neutron (SANS) and X-ray (SAXS) scattering. Experimental scattering curves of magnetosome samples, indicate the presence of polydisperse particles in the samples. The values of forward scattering intensities confirm the differences in average sizes of samples prepared by various methods. For the sonicated sample the coercive force is lower as a consequence of the existence partly individual particles. This fact causes the decrease of magnetocrystalline anisotropy and so changes in magnetic and hyperthermia properties. In consequence of this changes the energy loss and specific absorption rate are noticeable reduced in sonicated sample and thereby indicates variation in the relaxation process and heat distribution.

The Automated Device for Studying the Acoustic and Thermophysical Properties of Liquids

VERVEYKo Vyacheslav N., verveykovn@mail.ru Verveyko Marina V., Verveyko Darya V. Verisokin Andrey YU., Chebrov Nikita S.

Research Center for Condensed Matter Physics Kursk State University

Radishcheva 33, 305000 Kursk, Russia

The existing devices for studying the acoustic and thermophysical properties of substances have a number of drawbacks such as the excitation of the piezoelectric transducer by shock rectangular pulses which can lead to distortion of the signal emitted by the piezoelectric transducer or by continuous high frequency signal whereby the piezoelectric transducer is not in the free vibration mode but it is in forced vibration mode; a large number of functional blocks.

The authors have created the electronic block of generating, receiving and processing of the signals based on programmable logic integrated scheme (PLIS). The block operation control, the ultrasound speed measurements and calculation of thermophysical values carried out by means software installed on a personal computer. Visual observation and monitoring of the signals is carried out on a computer screen.

Increasing of action speed, accuracy and reliability of measurements achieved by using of modern high-speed electronic components and by exciting of piezoelectric transducer through packages of sinusoidal pulses that allows it to oscillate at the natural resonance frequency with the maximum possible amplitude without distortion of the signal.

This work was supported RFBR, project number 16-0801203.

$$
\star \star \star \star
$$

\section{XLVth Jubilee Winter School on Environmental Acoustics and Vibroacoustics}

\section{Acoustic Screens in Open Plan Rooms}

MikUlski Witold, wimik@ciop.pl

Central Institute for Labour Protection

- National Research Institute

Czerniakowska 16, 00-701 Warszawa, Poland

Among the open plan rooms, the petitioners' rooms can be distinguished. In these rooms many peoples talk simultaneously, being the noise source to each other. In order to ensure the privacy and the speech intelligibility in the open plan room, an appropriate acoustic treatment needs to be provided. The criteria are specified in: $\mathrm{PN}-$ B-02151-4 and EN ISO 3382-3. The acoustic properties of the open plan rooms are characterized by six parameters. Ensuring of the specific values of these parameters leads to a positive evaluation of acoustic criteria for open plan rooms.

In this paper, the influence of the height and the soundabsorbing parameters of the acoustic screens on the considered parameters are evaluated in order to find screen that ensure positive assessment of the acoustic properties of these room. The study was performed by numerical computations in ODEON software for the real open plan room. The results showed that it is possible to achieve appropriate acoustic properties of the open plan room, when the acoustic screen height and sound absorption coefficient are $1.8 \mathrm{~m}$ and 0.95 .

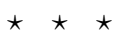

Method for Determining the Sound Power Level of Noise Sources in the Frequency Range Above $10 \mathrm{kHz}$

MikULSKI Witold, wimik@ciop.pl

Central Institute for Labour Protection

- National Research Institute

Czerniakowska 16, 00-701 Warszawa, Poland

This paper presents a method of the measurement of the sound power level of noise sources in the frequency 
range of $10-40 \mathrm{kHz}$. The method was developed on the basis of three other methods: the one developed by W. MiKuLSKI and J. RADOSZ in 2009 and the two specified in EN ISO 3744 and EN ISO 9295. Presented method allows to determine the sound power level by the measurement of sound pressure level over a reflecting plane and a hemispherical measuring surface. An amendment resulting from the attenuation of sound through the air is taken into account. The number of measuring points in this method is twice as used in the existing measurement standards (conducted a study). The number of circle scanning surfaces in this method is smaller than in the previous measurement standard (also examined). The method can be used when the declaration of noise emission by sources in the frequency range so far not taken into account, i.e. $10-40 \mathrm{kHz}$.

\section{Recommendations for Wind Turbine Noise Exposure Reduction at Workplaces Wind Farms}

Pleban Dariusz, daple@ciop.pl Augustýnska Danuta

Central Institute for Labour Protection

- National Research Institute

Czerniakowska 16, 00-701 Warszawa, Poland

The development of wind energy in Poland is accompanied by an increase in the number of people employed in the workplaces of wind energy sector. According to recent estimates, 8400 persons were employed in wind energy sector, with 600 of them directly employed in wind farms. Therefore, noise and infrasonic noise measurements at workplaces in the wind farm were carried out during service and maintenance activities carried out by the staff. The scope of the measurements at workplaces included the determination of the following parameters: the A-weighted sound pressure levels, the A-weighted maximum sound pressure levels, the $\mathrm{C}$-weighted peak sound pressure levels and the G-weighted sound pressure levels.

Besides, noise annoyance at workplaces was conducted by means of the questionnaire. The results of noise and infrasonic measurements and of surveys made it possible to lay down recommendations for wind turbine noise reduction at workplaces in wind farms.

$$
\star \star \star
$$

Noise at Selected Workplaces at the Packaging Manufacturing Plant

Smagowska Bożena, bosma@ciop.pl

Central Institute for Labour Protection

- National Research Institute

Czerniakowska 16, 00-701 Warszawa, Poland

The packaging market in Poland accounts for approx. $1.4 \%$ of the global packaging market and is steadily growing. According to data of the Central Statistical Office (GUS), there are approx. 8 thousand packaging manufacturing plants in Poland. The majority of them are small and medium-sized companies producing packaging made of wood, plastic; paper and cardboard; metal, glass and textiles. The main sources of noise emitted at the packaging manufacturing plants are (depending on the used technology): punching machines, cutters, folding presses and heat presses, foaming machines, shakers, and machines for the production of foams and regranulation of waste. This article presents the preliminary results of the measurement and assessment of exposure to noise carried out at selected workplaces at the packaging manufacturing plant. 